Internat. J. Math. \& Math. Sci.

Vol. 23, No. 12 (2000) 807-814

S0161171200002933

(C) Hindawi Publishing Corp.

\title{
HYPERINVARIANT SUBSPACES FOR SOME OPERATORS ON LOCALLY CONVEX SPACES
}

\author{
EDVARD KRAMAR
}

(Received 27 January 1999)

\begin{abstract}
Some results concerning hyperinvariant subspaces of some operators on locally convex spaces are considered. Denseness of a class of operators which have a hyperinvariant subspace in the algebra of locally bounded operators is proved.
\end{abstract}

Keywords and phrases. Locally convex space, hyperinvariant subspace, operator.

2000 Mathematics Subject Classification. Primary 47A15, 47B99, 46A32.

1. Introduction. Let $X$ be a locally convex space over the complex field $\mathbb{C}$. Each system of seminorms $P$ inducing its topology will be called a calibration. We denote by $\mathscr{P}(X)$ the collection of all calibrations on $X$ and by $\Lambda(X)$ all continuous seminorms with respect to the given topology. Let us denote by $\mathscr{L}(X)$ the set of all linear continuous operators on $X$ and by $\mathscr{K}(X)$ the set of compact operators on $X$, i.e., $T \in \mathscr{K}(X)$ if there exists a neighborhood $U$ such that $T(U)$ is a relatively compact set. We shall denote by $\operatorname{im}(T)$ the range of $T$ and by ker $T$ the null space of $T$. For a given $P \in \mathscr{P}(X)$, let $P=\left\{p_{\alpha}\right.$ : $\alpha \in \Delta\}$, where $\Delta$ is some index set. Choose any $\alpha \in \Delta$ and let $X_{\alpha}:=X / \operatorname{ker}\left(p_{\alpha}\right)$ denote the quotient space which is a normed space with respect to the norm $\left\|x_{\alpha}\right\|_{\alpha}=p_{\alpha}(x)$ where $x_{\alpha}=x+\operatorname{ker}\left(p_{\alpha}\right)$. The completeness of $X_{\alpha}$ we denote by $\tilde{X}_{\alpha}$. It is well known that for the dual spaces the following relation holds $X^{\prime}=\bigcup\left\{\left(\tilde{X}_{\alpha}\right)^{\prime}, \alpha \in \Delta\right\}$ (Floret and Wloka [2]). Let $B$ be an absolutely convex and bounded set, then $X_{B}:=\bigcup\{n B, n \in \mathbb{N}\}$ is a normed subspace in $X$ with respect to the norm $\|x\|_{B}:=\inf \{\lambda>0: x \in \lambda B\}$. It is easy to see that for any $p_{\alpha} \in P$ there is some $\lambda_{\alpha} \geq 0$ such that

$$
p_{\alpha}(x) \leq \lambda_{\alpha}\|x\|_{B}, \quad x \in X_{B} .
$$

A linear operator $T$ on $X$ is called nuclear if it can be written in the form

$$
T x=\sum_{j=1}^{\infty} \lambda_{j} c_{j}(x) a_{j}, \quad x \in X,
$$

where $\left(c_{j}\right)$ is an equicontinuous sequence in $X^{\prime},\left(a_{j}\right)$ is a sequence contained in an absolutely convex bounded set $B$ in $X$, such that $X_{B}$ is complete and $\left(\lambda_{j}\right) \in l_{1}$ (cf. Litvinov [8]). It is easy to see that the family of nuclear operators is an ideal in $\mathscr{L}(X)$ and that each nuclear operator is also compact. A linear operator $T$ on $X$ is called locally bounded, or $T \in \mathscr{L B}(X)$, if there exists a neighborhood $U$ such that $T(U)$ is bounded (see Uss [10]). Clearly, if $X$ is a normed space, then $\mathscr{L} B(X)=\mathscr{L}(X)$. 
For a given $P \in \mathscr{P}(X)$ we denote by $B_{P}(X)$ the algebra of universally bounded operators on $X$, i.e., the collection of all linear operators $T$ on $X$ for which

$$
p_{\alpha}(T x) \leq C p_{\alpha}(x), \quad x \in X, p_{\alpha} \in P
$$

where $C \geq 0$ is independent of $p_{\alpha} \in P$. The algebra $B_{P}(X)$ is a unital normed algebra with respect to the norm

$$
\|T\|_{P}=\sup \left\{p_{\alpha}(T x): p_{\alpha}(x) \leq 1, x \in X, p_{\alpha} \in P\right\} .
$$

LEMMA 1.1. Let $X$ be a Hausdorff locally convex space and $T \in \mathscr{L B}(X)$, then there exists a calibration $P^{\prime} \in \mathscr{P}(X)$ such that $T \in B_{P^{\prime}}(X)$.

Proof. Choose any calibration $P \in \mathscr{P}(X)$. Then there exists a neighborhood $U_{\gamma}$ such that $T\left(U_{\gamma}\right)$ is bounded. Without loss of generality we may assume that $U_{\gamma}$ is the open semiball corresponding to the seminorm $p_{\gamma} \in P$. For any $p_{\alpha} \in P$ there is some $C_{\alpha} \geq 0$ such that $\sup \left\{p_{\alpha}(T x): x \in U_{\gamma}\right\} \leq C_{\alpha}$. Hence for $x \in X$ for which $p_{\gamma}(x)<1$ it follows $p_{\alpha}(T x) \leq C_{\alpha}$. Let us prove that

$$
p_{\alpha}(T x) \leq C_{\alpha} p_{\gamma}(x), \quad p_{\alpha} \in P, x \in X .
$$

Fix any $p_{\alpha} \in P$ and assume first that $C_{\alpha}>0$. If for some $z \in X$ there is $p_{\alpha}(T z)>$ $C_{\alpha} p_{\gamma}(z)$ then there exists $\mu>0$ such that $p_{\alpha}(T z)>\mu>C_{\alpha} p_{\gamma}(z)$ and then for the vector $\left(C_{\alpha} / \mu\right) z$ we have a contradiction with the above implication. If $C_{\alpha}=0$ then (1.5) holds with zero on both sides. Especially, we have $p_{\gamma}(T x) \leq C_{\gamma} p_{\gamma}(x), x \in X$. Let us define $P^{\prime}=\left\{p_{\alpha}^{\prime}: p_{\alpha} \in P\right\}$, where $p_{\alpha}^{\prime}(x)=\max \left\{p_{\alpha}(x), C_{\alpha} p_{\gamma}(x)\right\}, x \in X$. We readily verify that $P^{\prime}$ is again a calibration. Now, we can for any $p_{\alpha}^{\prime} \in P^{\prime}$ estimate

$$
p_{\alpha}^{\prime}(T x)=\max \left\{p_{\alpha}(T x), C_{\alpha} p_{\gamma}(T x)\right\} \leq C_{\alpha} C_{0} p_{\gamma}(x) \leq C_{0} p_{\alpha}^{\prime}(x),
$$

where $C_{0}=\max \left\{1, C_{\gamma}\right\}$. Hence $T \in B_{P^{\prime}}(X)$.

By invariant subspace of $T \in \mathscr{L}(X)$ we mean a closed subspace $M \subset X$ with the property $T(M) \subset M$. Likewise, $M$ is hyperinvariant for $T$ if it is invariant for every operator $B \in \mathscr{L}(X)$ that commutes with $T$.

2. Main results. We consider some instances of the existence of a hyperinvariant subspace for some operators on locally convex spaces. We start with the following result obtained by Ma in [9] as a generalization from normed spaces (Kim et al. [4]) to locally convex spaces.

TheOrem 2.1. Let $X$ be a Hausdorff locally convex space, $T \in \mathscr{L}(X)$ and let $K \in$ $\mathscr{K}(X)$ be such that the commutator $T K-K T$ has rank at most one. Then $T$ has a nontrivial hyperinvariant subspace.

We consider two cases in which the operator has the above property. Denote $\mathbb{N}_{0}:=$ $\mathbb{N} \cup\{0\}$.

THEOREM 2.2. Let $X$ be a complete Hausdorff barreled locally convex space and $T \in \mathscr{L}(X)$ an invertible operator, let $a \in X, \varphi \in X^{\prime}$ and $P \in \mathscr{P}(X)$ be such that: 
(i) $\left\{T^{n} a: n \in \mathbb{N}_{0}\right\} \subset B$, for some absolutely convex bounded set $B$ in $X$ for which $X_{B}$ is complete,

(ii) there is some $p_{\gamma} \in P$, such that $\left(T^{\prime}\right)^{-n} \varphi \in X_{\gamma}^{\prime}$ for all $n \in \mathbb{N}_{0}$,

(iii) $\sum_{n=0}^{\infty}\left\|\left(T^{\prime}\right)^{-n} \varphi\right\|_{\gamma}\left\|T^{n} a\right\|_{B}<\infty$.

Then there exists a nontrivial hyperinvariant subspace for $T$.

Proof. Since $T$ is invertible, the adjoint operator $T^{\prime}$ is invertible, too. Define an operator sequence as follows

$$
K_{n}=\sum_{k=0}^{n} T^{k} a \otimes\left(\left(T^{\prime}\right)^{-k} \varphi\right), \quad n \in \mathbb{N} .
$$

Let $q_{\alpha}^{M}$ be a member of a calibration inducing the topology $\tau_{b}$ on $\mathscr{L}(X)$ of the uniform convergence on bounded subsets of $X$, where $M$ is a bounded set on $X$ and $q_{\alpha}^{M}$ corresponds to $M$ and some $p_{\alpha} \in P$. Let $m>n, m, n \in \mathbb{N}$, then we can estimate

$$
\begin{aligned}
q_{\alpha}^{M}\left(K_{m}-K_{n}\right)=\sup _{x \in M} p_{\alpha}\left(K_{m} x-K_{n} x\right) & \leq \sum_{k=n+1}^{m} \sup _{x \in M} p_{\alpha}\left[\left(T^{\prime}\right)^{-k} \varphi(x) T^{k} a\right] \\
& \leq \sum_{n+1}^{m}\left\|\left(T^{\prime}\right)^{-k} \varphi\right\|_{\gamma} \sup _{x \in M} p_{\gamma}(x) p_{\alpha}\left(T^{k} a\right) \\
& \leq \lambda_{\alpha} \cdot \sup _{x \in M} p_{\gamma}(x) \sum_{n+1}^{m}\left\|\left(T^{\prime}\right)^{-k} \varphi\right\|_{\gamma}\left\|T^{k} a\right\|_{B}
\end{aligned}
$$

and by (iii), $\left\{K_{n}\right\}$ is a $\tau_{b}$-Cauchy sequence on $\mathscr{L}(X)$. Since $X$ is barreled and complete, $\mathscr{L}(X)$ is quasicomplete with respect to $\tau_{b}$ (Köthe [6]) and hence also sequentially complete. Thus, the sequence $\left\{K_{n}\right\}$ converges to some operator $K \in \mathscr{L}(X)$. Denote by $J_{B}$ the injection map of $X_{B}$ into $X$ and by $\pi_{\gamma}$ the natural map $X \mapsto X_{\gamma}$. Then $K=J_{B} \hat{K}_{\gamma} \pi_{\gamma}$, where $\widehat{K}_{\gamma}$ can be written in the form

$$
\hat{K}_{\gamma} x_{\gamma}=\sum_{k=0}^{\infty} \psi_{k}^{\gamma}\left(x_{\gamma}\right) a_{k},
$$

where $\psi_{k}^{\gamma}\left(x_{\gamma}\right)=\left(\left(T^{\prime}\right)^{-k} \varphi\right)(x)$ for $x_{\gamma}=x+\operatorname{ker}\left(p_{\gamma}\right)$ and $a_{k}=T^{k} a, k \in \mathbb{N}_{0}$. Taking into account the above assumptions, the operator $\hat{K}_{\gamma}$ acting between two normed spaces is nuclear, and since $J_{B}$ and $\pi_{\gamma}$ are continuous, $K$ is nuclear and then also compact [2]. It is not hard to verify that $T K-K T=-a \otimes T^{\prime} \varphi$, thus, it has rank one and applying Theorem 2.1, the proof is complete.

Corollary 2.3. Let $X$ be a complete barreled Hausdorff locally convex space, $T \in$ $\mathscr{L}(X)$ and $C \in \mathscr{L}(X)$ an invertible operator such that $C \in\{T\}^{\prime \prime}$ (in the second commutant) and suppose that there exist $a \in X, \varphi \in X^{\prime}$ and $P \in \mathscr{P}(X)$ such that:

(i) $\left\{C^{n} a: n \in \mathbb{N}_{0}\right\} \subset B$ for some absolutely convex bounded set $B$ in $X$ for which $X_{B}$ is complete,

(ii) there is some $p_{\gamma} \in P$, such that $\left(C^{\prime}\right)^{-n} \varphi \in X_{\gamma}^{\prime}$, for all $n \in \mathbb{N}_{0}$,

(iii) $\sum_{n=0}^{\infty}\left\|\left(C^{\prime}\right)^{-n} \varphi\right\|_{\gamma}\left\|C^{n} a\right\|_{B}<\infty$.

Then $T$ has a nontrivial hyperinvariant subspace. 
Proof. By the above theorem, $C$ has a nontrivial hyperinvariant subspace and since $\{T\}^{\prime} \subset\{C\}^{\prime}$, the operator $T$ has a nontrivial hyperinvariant subspace, too.

As in Kim et al. [5] we can prove for locally convex spaces the following theorem.

THEOREM 2.4. Let $X$ be a Hausdorff locally convex space and $T \in \mathscr{L}(X)$ a nonscalar operator. If $T$ or $T^{\prime}$ has an eigenvector, then $T$ has a nontrivial hyperinvariant subspace.

Proof. Let $T z=\lambda z, z \neq 0$, and choose $\varphi \in X^{\prime}$ such that $(T-\lambda I)^{\prime} \varphi \neq 0$. Then for $K:=z \otimes \varphi$ we have $T K-K T=z \otimes(\lambda I-T)^{\prime} \varphi$ and by Theorem 2.1 the conclusion follows. If $T^{\prime}$ has an eigenvector the proof is similar.

For a given $T \in \mathscr{L}(X)$, the number $\lambda \in \mathbb{C}$ is in the resolvent set of $T$, or $\lambda \in \varrho(T)$, if and only if there exists $(T-\lambda I)^{-1} \in \mathscr{L}(X)$. The spectrum is the set $\sigma(T)=\mathbb{C} \backslash \varrho(T)$. As in a normed space we can define the following main subsets of the spectrum: $\sigma_{p}(T)$, $\sigma_{c}(T)$, and $\sigma_{r}(T)$-the point spectrum, the continuous, and the residual spectrum, respectively, as follows (see Uss [10]): $\lambda \in \sigma_{p}(T)$ if and only if $T-\lambda I$ is not one-to-one, $\lambda \in \sigma_{r}(T)$ if and only if $\lambda \notin \sigma_{p}(T)$ and $\overline{\operatorname{im}(T-\lambda I)} \neq X$ and $\lambda \in \sigma_{c}(T)$ if and only if $\lambda \notin\left(\sigma_{p}(T) \cup \sigma_{r}(T)\right)$ and $(T-\lambda I)^{-1}$ is not continuous. When $X$ is complete, then $\sigma(T)=\sigma_{p}(T) \cup \sigma_{r}(T) \cup \sigma_{c}(T)$ (see [10]).

THEOREM 2.5. Let $X$ be a complete Hausdorff locally convex space. Then the set of all continuous linear operators which have a nontrivial hyperinvariant subspace is $\tau_{b}$-dense in $\mathscr{L} B(X)$.

Proof. We may assume that $X$ is not normable since for normed spaces such a result holds (see Kim et al. [5]). Choose any nonzero $T \in \mathscr{L} B(X)$. Clearly, $T \neq \mu I$, since $I \notin \mathscr{L B}(X)$. Let $P=\left\{p_{\alpha}: \alpha \in \Delta\right\}$ be a calibration on $X$. As in the proof of Lemma 1.1 there is some $p_{\gamma} \in P$ such that for any $p_{\alpha} \in P$ there is some $C_{\alpha}>0$ such that (1.5) holds. By Uss [10], $\sigma(T) \neq \varnothing$, so, we can choose some $\lambda \in \sigma(T)$. If $\lambda \in \sigma_{p}(T)$, then $T$ has, by Theorem 2.4, a hyperinvariant subspace. For $\lambda \notin \sigma_{p}(T)$ we still have two cases. If $\lambda \in \sigma_{r}(T)$, then $\overline{\operatorname{im}(T-\lambda I)} \neq X$ and, by the Hahn-Banach theorem, there exists a nontrivial $\varphi \in X^{\prime}$ such that $\varphi((T-\lambda I) x)=0$ for all $x \in X$. Consequently, $\lambda \in \sigma_{p}\left(T^{\prime}\right)$ and by Theorem 2.4, $T$ has a nontrivial hyperinvariant subspace. Let $\lambda \in \sigma_{c}(T)$. Then there exists a net $\left(a_{\delta}\right)_{\delta \in \Gamma}$ such that $(T-\lambda I) a_{\delta} \rightarrow 0$ and $a_{\delta} \rightarrow 0$. First, suppose that $\lambda \neq 0$ and prove that $p_{\gamma}\left(a_{\delta}\right) \nrightarrow 0$. For any $p_{\alpha} \in P$ we have

$$
|\lambda| p_{\alpha}\left(a_{\delta}\right) \leq p_{\alpha}\left((\lambda I-T) a_{\delta}\right)+p_{\alpha}\left(T a_{\delta}\right) \leq p_{\alpha}\left((\lambda I-T) a_{\delta}\right)+C_{\alpha} p_{\gamma}\left(a_{\delta}\right) .
$$

If $p_{\gamma}\left(a_{\delta}\right) \rightarrow 0$, then $p_{\alpha}\left(a_{\delta}\right) \rightarrow 0$ for all $\alpha \in \Delta$, hence $a_{\delta} \rightarrow 0$ which is a contradiction. So, we may assume that

$$
p_{\gamma}\left(a_{\delta}\right) \geq 1, \quad \delta \in \Gamma .
$$

Now, let $\lambda=0$. If for some $\delta^{\prime} \in \Gamma: p_{\gamma}\left(a_{\delta^{\prime}}\right)=0$, then by (1.5), $p_{\alpha}\left(T a_{\delta^{\prime}}\right)=0$ for all $\alpha \in \Delta$, and consequently $T a_{\delta^{\prime}}=0$, which is a contradiction with $\lambda \notin \sigma_{p}(T)$. Thus, $p_{\gamma}\left(a_{\delta}\right) \neq 0$ for all $\delta \in \Gamma$ and we may again assume that (2.5) holds. Now, for each $\delta \in \Gamma$ by the Hahn-Banach theorem, there exists some $\varphi_{\delta} \in X^{\prime}$ such that

$$
\varphi_{\delta}\left(a_{\delta}\right)=p_{\gamma}\left(a_{\delta}\right), \quad\left|\varphi_{\delta}(x)\right| \leq p_{\gamma}(x), \quad x \in X .
$$


Any $x \in X$ can be written in the form

$$
x=\frac{\varphi_{\delta}(x)}{p_{\gamma}\left(a_{\delta}\right)} a_{\delta}+z_{\delta}, \quad \delta \in \Gamma,
$$

where $z_{\delta} \in \operatorname{ker}\left(\varphi_{\delta}\right)$. Define an operator net $\left(T_{\delta}\right)_{\delta \in \Gamma}$ as follows

$$
T_{\delta} x:=\frac{\varphi_{\delta}(x)}{p_{\gamma}\left(a_{\delta}\right)} \lambda a_{\delta}+T z_{\delta}, \quad x \in X, \delta \in \Gamma .
$$

Let us take any $p_{\alpha} \in P$ and any bounded set $M$ on $X$ which generate one of the seminorms $q_{\alpha}^{M}$ of a calibration defining the topology $\tau_{b}$. Then we can estimate

$$
\begin{aligned}
q_{\alpha}^{M}\left(T_{\delta}-T\right)=\sup _{x \in M} p_{\alpha}\left(\left(T_{\delta}-T\right) x\right) & \leq \sup _{x \in M} \frac{\left|\varphi_{\delta}(x)\right|}{p_{\gamma}\left(a_{\delta}\right)} p_{\alpha}\left(T a_{\delta}-\lambda a_{\delta}\right) \\
& \leq \sup _{x \in M} p_{\gamma}(x) p_{\alpha}\left(T a_{\delta}-\lambda a_{\delta}\right),
\end{aligned}
$$

where the right-hand side tends to zero. So, the difference $T_{\delta}-T$ is $\tau_{b}$-convergent to zero and since $\lambda \in \sigma_{p}\left(T_{\delta}\right)$, by Theorem 2.4 all operators $T_{\delta}$ have a nontrivial hyperinvariant subspace.

In the sequel, we shall generalize another result from Kim et al. [3] to locally convex spaces. In the proof, one needs some lemmas which we shall write down for such spaces.

A linear operator $T \in \mathscr{L}(X)$ is said to be bounded below if for any seminorm $p \in \Lambda(X)$ there is some $q \in \Lambda(X)$ such that

$$
q(T x) \geq p(x), \quad x \in X .
$$

LEMMA 2.6. Let $X$ be a complete Hausdorff locally convex space and $T \in \mathscr{L}(X)$. Then $T$ is invertible in $\mathscr{L}(X)$ if and only if it is bounded below and $\overline{\operatorname{im}(T)}=X$.

Proof. If $T$ is invertible then $\operatorname{im}(T)=X$ and $T^{-1}$ is continuous, thus $T$ is bounded below. Conversely, let $T$ be bounded below with dense range. Let us prove that $\operatorname{im}(T)$ is closed. Take any convergent net $\left(y_{\delta}\right)_{\gamma \in \Gamma} \subset \operatorname{im}(T), y_{\delta} \rightarrow y$ and let $y_{\delta}=T x_{\delta}, x_{\delta} \in$ $X, \delta \in \Gamma$. Then by (2.10) it follows that $\left(x_{\delta}\right)$ is a Cauchy net and thus it is convergent $x_{\delta} \rightarrow x$. By continuity of $T$ it follows that $T x_{\delta} \rightarrow T x$ hence $y \in \operatorname{im}(T)$. The range is closed and dense, hence $\operatorname{im}(T)=X$.

In the vector space $\tilde{X}:=X \times X$ we define the topology $\tilde{\tau}$ by the following system of seminorms $\tilde{P}=\{\tilde{p}\}$, where $\tilde{p}(\tilde{x})=p_{1}\left(x_{1}\right)+p_{2}\left(x_{2}\right), p_{1}, p_{2} \in P$ for some $P \in \mathscr{P}(X)$ and $\tilde{x}=\left(x_{1}, x_{2}\right) \in \tilde{X}$. Let us denote by $\Lambda(\tilde{X})$ all continuous seminorms on $\tilde{X}$. It is easy to see that $\tilde{X}$ is complete whenever $X$ is complete. Let us define $(A \oplus B)(x, y):=(A x, B y)$.

LEMMA 2.7. Let $X$ be a complete Hausdorff locally convex space and $A_{1}, A_{2} \in \mathscr{L}(X)$. Then the operator $\tilde{A}:=A_{1} \oplus A_{2}$ is invertible in $\mathscr{L}(\tilde{X})$ if and only if $A_{1}$ and $A_{2}$ are invertible in $\mathscr{L}(X)$.

Proof. Let us suppose that $A_{i}, i=1,2$, are invertible. Then it is easy to see that for $\tilde{A}$ the inverse operator is $A_{1}^{-1} \oplus A_{2}^{-1}$. Let $\tilde{A}$ be invertible, then it is bounded below. 
For any $\tilde{p} \in \Lambda(\tilde{X})$ there is some $\tilde{q} \in \Lambda(\tilde{X})$ such that $\tilde{q}(\tilde{A} \tilde{x}) \geq \tilde{p}(\tilde{x}), \tilde{x} \in \tilde{X}$. Writing $\tilde{p}=p_{1}+p_{2}$ and $\tilde{q}=q_{1}+q_{2}$, with $p_{1}, p_{2}, q_{1}, q_{2} \in \Lambda(X)$ we have

$$
q_{1}\left(A_{1} x_{1}\right)+q_{2}\left(A_{2} x_{2}\right) \geq p_{1}\left(x_{1}\right)+p_{2}\left(x_{2}\right), \quad \tilde{x}=\left(x_{1}, x_{2}\right) \in \tilde{X} .
$$

Replacing $\tilde{x}$ once with $(x, 0)$ and then by $(0, x)$ we obtain

$$
q_{1}\left(A_{1} x\right) \geq p_{1}(x), \quad q_{2}\left(A_{2} x\right) \geq p_{2}(x), \quad x \in X .
$$

Since $\tilde{p}$ is arbitrary so are $p_{1}$ and $p_{2}$ which means that $A_{1}$ and $A_{2}$ are bounded below. By invertibility of $\tilde{A}$ in $\mathscr{L}(\tilde{X})$ there exists the operator $\left(\tilde{A}^{\prime}\right)^{-1}$ which is equal to $\left(\tilde{A}^{-1}\right)^{\prime}$ and it is continuous in the strong topology in $\mathscr{L}\left(\tilde{X}^{\prime}\right)$ (see [2]), where $\tilde{A}^{\prime}=A_{1}^{\prime} \oplus A_{2}^{\prime}$. Thus, $\tilde{A}^{\prime}$ is bounded below. In the same manner as above, $A_{1}^{\prime}$ and $A_{2}^{\prime}$ are then bounded below and hence injective. By the relations $\overline{\operatorname{im}\left(A_{i}\right)}=\operatorname{ker}\left(A_{i}^{\prime}\right)^{\perp}, i=1,2$ (Köthe [6]) the operators $A_{1}$ and $A_{2}$ have dense range. By Lemma 2.6 both are invertible.

LEMмA 2.8. Let $X$ be a Hausdorff locally convex space, $T \in \mathscr{L B}(X)$ and $\left\{K_{n}\right\} a$ sequence of operators in $B_{P}(X)$ for some $P \in \mathscr{P}(X)$ and such that $\left\|K_{n}\right\|_{P} \rightarrow 0$. Let $K_{0} \in \mathscr{K}(X)$ and

$$
K_{n} T=T K_{n+1} \quad \text { or } \quad K_{n+1} T=T K_{n}, \quad n=0,1,2, \ldots
$$

Then $T$ has a nontrivial hyperinvariant subspace.

The proof with the first assumption in (2.13) is made by Kramar [7], the proof with the second one is similar. In the next lemma one needs the Riesz functional calculus which can be generalized to locally convex spaces for operators of the form $T=$ $\alpha I+T_{1}$, where $\alpha \in \mathbb{C}$ and $T_{1}$ is a locally bounded operator (see Uss [10]).

LEMMA 2.9. Let $X$ be a complete barreled Hausdorff locally convex space, $T \in \mathscr{L}(X)$ a nonscalar operator, $K \in \mathscr{K}(X)$ a nonzero operator and $f$ an analytic function on an open neighborhood $U$ of $\sigma(K)$ and such that $K T=T f(K)$. Let $f(0)=0$ and $\sigma_{p}(T)=$ $\sigma_{p}\left(T^{\prime}\right)=\varnothing$. Then $f$ maps $\sigma(T)$ onto itself and for every integer $k$, the function $f^{k}$ has no fixed point on $\sigma(K)$ except zero and the following relations hold

$$
f^{n}(K) T=T f^{n+1}(K), \quad K T^{n}=T^{n} f^{n}(K), \quad n \in \mathbb{N} .
$$

In the proof one may restrict to nonnormable case, then for $K \in \mathscr{K}(X)$ we have $\{0\} \subset \sigma(K)$ (see [10]). The proof is then similar to that one in normed space using Lemma 2.7 and the following facts in locally convex setting:

(i) if $\lambda \neq 0$ and $K \in \mathscr{K}(X)$ then $\lambda \in \sigma_{p}(K)$ if and only if $\lambda \in \sigma_{p}\left(K^{\prime}\right)$ (see Edwards [1]),

(ii) if $K_{1}$ and $K_{2}$ are locally bounded then $K_{1} \oplus K_{2}$ is locally bounded too,

(iii) the Riesz functional calculus and spectral mapping theorem for locally bounded operators (see Uss [10]).

LEMMA 2.10. Let $X$ be a complete barreled Hausdorff locally convex space, $K, T, f$, and $U$ as in the previous lemma. Then $\sigma(K)=\{0\}$ if either $\left|f^{\prime}(0)\right|<1$ or $\left|f^{\prime}(0)\right|>1$ and $\operatorname{ker}(K)=\{0\}$.

The proof is the same as in normed case using the above-mentioned facts. 
THEOREM 2.11. Let $X$ be a complete barreled Hausdorff locally convex space, $T \in \mathscr{L}(X)$ a nonscalar and $K \in \mathscr{K}(X)$ a nonzero operator, $f$ an analytic function on an open neighborhood $U$ of the spectrum $\sigma(K)$ such that $K T=T f(K)$. Then $T$ has a nontrivial hyperinvariant subspace whether one of the following conditions holds

(i) $\left|f^{\prime}(0)\right|<1$,

(ii) $\left|f^{\prime}(0)\right|>1$ and $\sigma(K)=\{0\}$,

(iii) $\left|f^{\prime}(0)\right|>1$ and $\operatorname{ker}(K)=\{0\}$.

Proof. As in normed case, we may assume that $f(0)=0$ and that $\sigma_{p}(T)=\sigma_{p}\left(T^{\prime}\right)=$ $\varnothing$ (Theorem 2.4) and in all three cases by Lemma 2.10 it follows that $\sigma(K)=\{0\}$. Let us treat the case $\left|f^{\prime}(0)\right|<1$, then there are $0<c<1$ and $\delta>0$ such that $|f(\zeta)|<c|\zeta|$ for $0<|\zeta|<\delta$. Define a sequence of operators: $K_{0}=K$ and $K_{n}=f^{n}\left(K_{0}\right), n \in \mathbb{N}$. Let us prove that for this sequence the assumptions of Lemma 2.8 are satisfied. Choose $\varepsilon \in(0, \delta)$ and denote $S_{\varepsilon}=\{\lambda, \lambda \in \mathbb{C},|\lambda|=\varepsilon\}$ then we may use the functional calculus (see Uss [10]) in the following manner

$$
\begin{aligned}
K_{n} & =\frac{1}{2 \pi i} \int_{S_{\varepsilon}} f^{n}(\lambda)(\lambda I-K)^{-1} d \lambda \\
& =\frac{1}{2 \pi i} \int_{S_{\varepsilon}} \frac{f^{n}(\lambda) I}{\lambda} d \lambda+\frac{1}{2 \pi i} \int_{S_{\varepsilon}} \frac{f^{n}(\lambda) K(\lambda I-K)^{-1}}{\lambda} d \lambda \\
& =f^{n}(0) I+\frac{1}{2 \pi i} \int_{S_{\varepsilon}} \frac{f^{n}(\lambda) K(\lambda I-K)^{-1}}{\lambda} d \lambda,
\end{aligned}
$$

where $f^{n}(0)=0$. For a given $P=\left\{p_{\alpha}\right\} \in \mathscr{P}(X)$ since $K \in \mathscr{K}(X)$ there is some semiball $U_{\gamma}$ such that $K\left(U_{\gamma}\right)$ is bounded and the set $(\lambda I-K)^{-1} K\left(U_{\gamma}\right)$ is for $\lambda \in S_{\varepsilon}$ bounded too. Fix any $p_{\alpha} \in P$, let $C_{\gamma, \alpha}^{\lambda}=\sup \left\{p_{\alpha}\left((\lambda I-K)^{-1} K x\right), x \in U_{\gamma}\right\}$ and $C_{\gamma, \alpha}=\sup \left\{C_{\gamma, \alpha}^{\lambda}, \lambda \in\right.$ $\left.S_{\varepsilon}\right\}$. Then as in the proof of Lemma 1.1 we have $p_{\alpha}\left((\lambda I-K)^{-1} K x\right) \leq C_{\gamma, \alpha} p_{\alpha}(x), x \in X$. If we define $P^{\prime}=\left\{p_{\alpha}^{\prime}\right\}$, where $p_{\alpha}^{\prime}=\max \left\{p_{\alpha}, C_{\gamma, \alpha} \cdot p_{\alpha}\right\}$ all operators $(\lambda I-K)^{-1} K$ are in $B_{P^{\prime}}(X)$ and

$$
p_{\alpha}^{\prime}\left((\lambda I-K)^{-1} K x\right) \leq M_{\gamma} p_{\alpha}^{\prime}(x), \quad x \in X, \lambda \in S_{\varepsilon}
$$

with $M_{\gamma}=\max \left\{1, C_{\gamma, \gamma}\right\}$. So, we can for each $p_{\alpha}^{\prime} \in P^{\prime}$ and $x \in X$ estimate

$$
p_{\alpha}^{\prime}\left(K_{n} x\right) \leq \sup _{\lambda \in S_{\varepsilon}} \frac{\left|f^{n}(\lambda)\right|}{|\lambda|} \cdot \sup _{\lambda \in S_{\varepsilon}} p_{\alpha}\left(K(\lambda I-K)^{-1} x\right) \cdot \varepsilon \leq \varepsilon c^{n} M_{\gamma} p_{\alpha}^{\prime}(x) .
$$

Hence $\left\|K_{n}\right\|_{P^{\prime}} \leq c^{n} \cdot M_{\gamma} \varepsilon$ and we have $\left\|K_{n}\right\|_{P^{\prime}} \rightarrow 0$. By Lemma 2.8 the operator $T$ has a nontrivial hyperinvariant subspace. In the case $\left|f^{\prime}(0)\right|>1$ the proof is similar. First, one can find a function $h$ analytic on a neighborhood $\mho_{1}$ of the origin such that $h(f(\zeta))=\zeta, \zeta \in U_{1}$. Then as above one can verify that all assumptions of Lemma 2.8 are satisfied for $h^{n}(K), n \in \mathbb{N}$ and the conclusion follows.

\section{REFERENCES}

[1] R. E. Edwards, Functional Analysis. Theory and Applications, Holt, Rinehart and Winston, New York, 1965. MR 36\#4308. Zbl 182.16101.

[2] K. Floret and J. Wloka, Einführung in die Theorie der Lokalkonvexen Räume, Lecture Notes in Mathematics, vol. 56, Springer-Verlag, Berlin, Heidelberg, New York, 1968. MR 37\#1945. Zbl 155.45101. 
[3] H. W. Kim, R. Moore, and C. M. Pearcy, A variation of Lomonosov's theorem, J. Operator Theory 2 (1979), no. 1, 131-140. MR 81b:47007. Zbl 433.47010.

[4] H. W. Kim, C. Pearcy, and A. L. Shields, Rank-one commutators and hyperinvariant subspaces, Michigan Math. J. 22 (1975), no. 3, 193-194 (1976). MR 52\#11626. Zbl 319.47027.

[5] - Sufficient conditions for rank-one commutators and hyperinvariant subspaces, Michigan Math. J. 23 (1976), no. 3, 235-243 (1977). MR 57\#3881. Zbl 346.47026.

[6] G. Köthe, Topological Vector Spaces. II, Springer-Verlag, Berlin, Heidelberg, New York, 1979. MR 81g:46001. Zbl 417.46001.

[7] E. Kramar, Invariant subspaces for some operators on locally convex spaces, Comment. Math. Univ. Carolin. 38 (1997), no. 4, 635-644. MR 99a:47013.

[8] G. L. Litvinov, On the traces of linear operators in locally convex spaces, Selecta Math. Soviet. 8 (1989), no. 3, 203-212. Zbl 686.47021.

[9] T. W. Ma, On rank one commutators and triangular representations, Canad. Math. Bull. 29 (1986), no. 3, 268-273. MR 87g:47028. Zbl 555.47004.

[10] P. Uss, Sur les opérateurs bornés dans les espaces localement convexes, Studia Math. 37 (1970/71), 139-158. MR 46\#2466. Zbl 212.15901.

Kramar: University of LJUblJana, DePartment of Mathematics, Jadranska 19, 1000 LJUBLJANA, SLOVENIA

E-mail address: Edvard.Kramar@fmf.uni-1j.si 


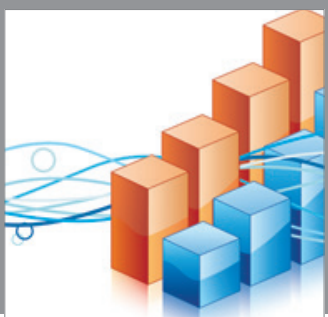

Advances in

Operations Research

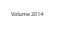

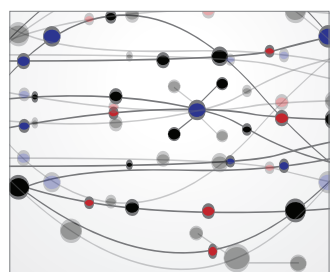

\section{The Scientific} World Journal
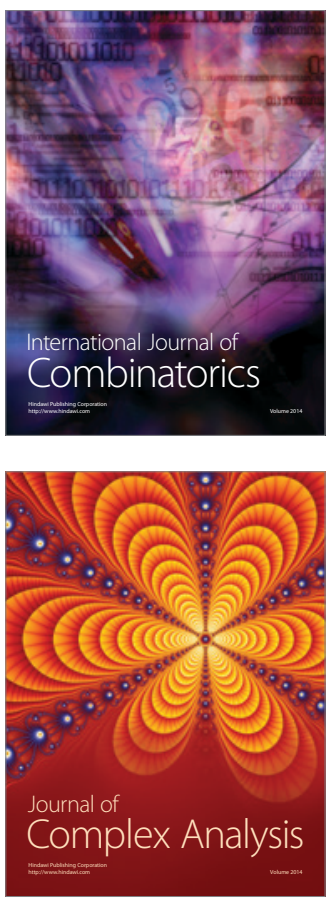

International Journal of

Mathematics and

Mathematical

Sciences
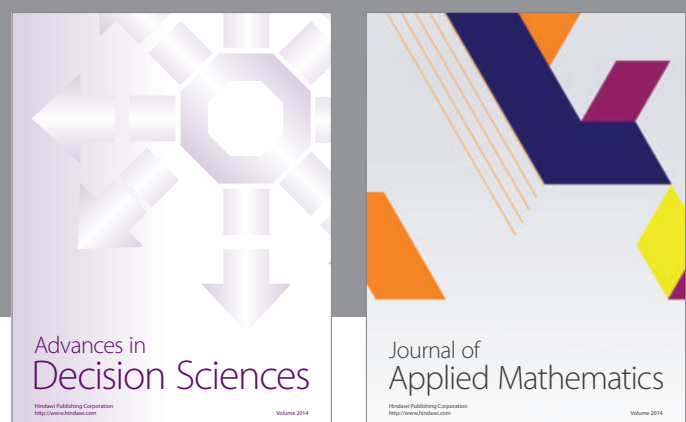

Journal of

Applied Mathematics
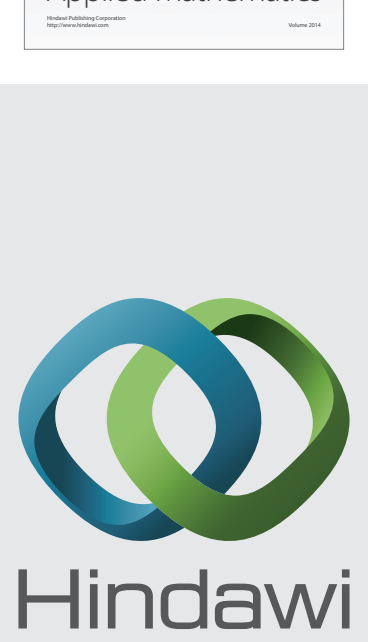

Submit your manuscripts at http://www.hindawi.com
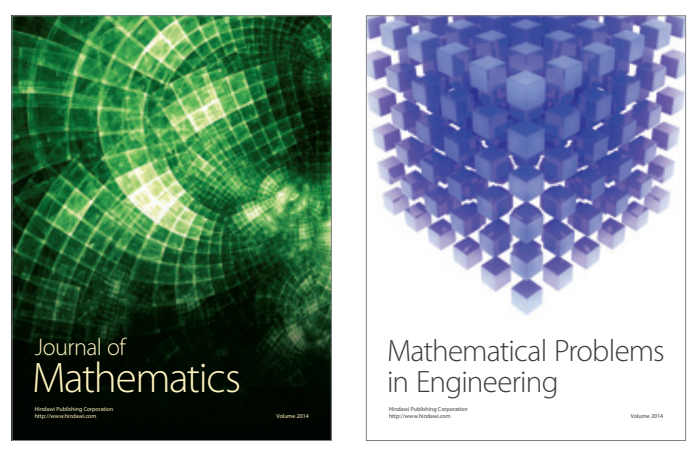

Mathematical Problems in Engineering
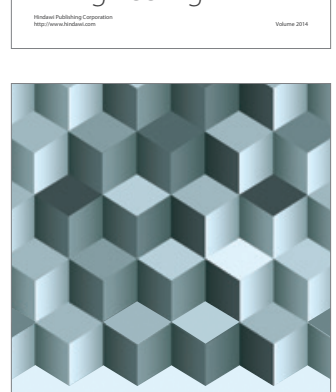

Journal of

Function Spaces
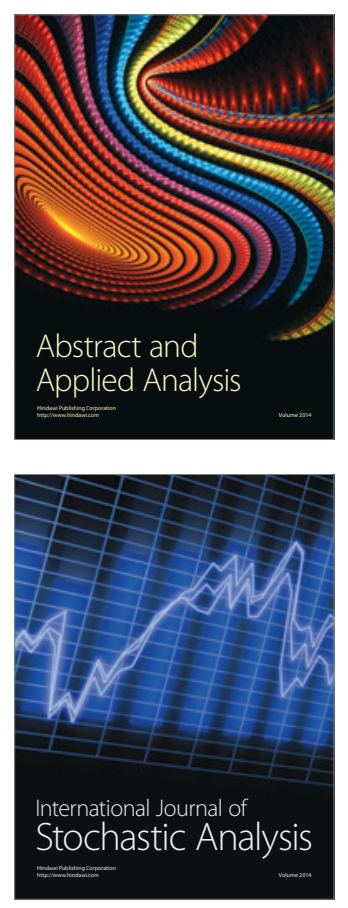

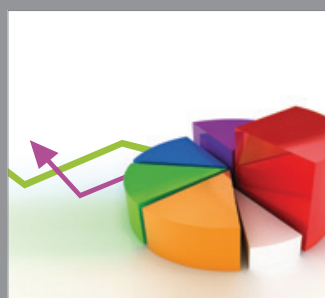

ournal of

Probability and Statistics

Promensencen
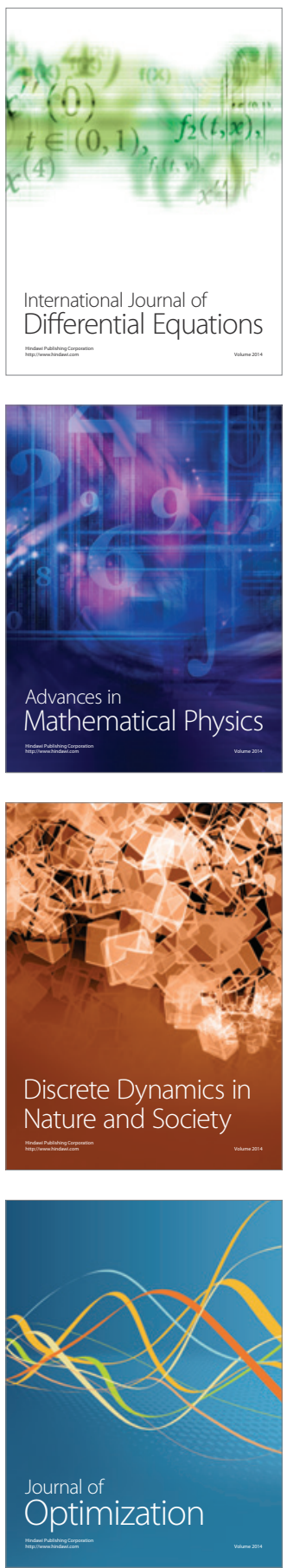